MODELING, IDENTIFICATION AND CONTROL, 2002, VOL. 23, NO. 2, 93-115

doi:10.4173/mic.2002.2.2

\title{
Simulation of a Hydrometallurgical Leaching Reactor Modeled as a DAE system*
}

\author{
M. DUEÑAS DÍEZ, G. AUSLANDł, M. FJELD§ and B. LIE† \\ Keywords: Population Balance Equation, Differential and Algebraic Equations, \\ leaching, dynamic modeling, ferrosilicon
}

An existing dynamic model of the main reactor in the Silgrain ${ }^{\text {Ri }}$ process for the production of $\mathrm{Si}$ from $\mathrm{FeSi}$ has been extended here in order to resemble more closely the behavior of the real reactor. The previous model was based on the application of macroscopic mass conservation law, the population balance equation and the assumptions of complete mixing and isothermic conditions. The major modifications are the inclusion of the condition governing the entrainment of particles in the outflow, and the formulation of the energy balance.

The extended model consists of 1 integrodifferential equation, 4 implicit ordinary differential equations, 8 algebraic equations and 3 integral equations. After discretization in the particle size space, a system of Differential and Algebraic Equations (DAE) is obtained. DAEs are not ODEs and they require analysis and characterization and may require reformulation. After such analysis, it was concluded that the system is implicit index-one for the usual range of operation and that a method based on the Backward Differentiation Formulas (BDF) can be used for its solution.

The model was implemented in MATLAB and the ode15s code was used for solving the system of equations. The simulation results are satisfactory and seem to match qualitatively with the known operation of the reactor. The model is suitable for further use in designing a model-based control scheme.

\section{Introduction}

Silicon (Si) is one of the most important technical materials due to its properties as semiconductor. Elkem Bremanger at Svelgen (Norway) produces Si metal via a patented hydrometallurgical leaching process called the Silgrain ${ }^{\mathbf{R} /}$ process. Hydrometallurgical leaching belongs to the category of reactive particulate processes, which are inherently more difficult to describe than reactive systems comprised of one or more bulk phases, due to the fact that dispersed particles in liquid phase are invariably polydispersed in nature.

A model of the leaching reactor in the Silgrain process was developed in Dueñas and Lie (2000) to simulate the dynamic response of the main reactor in the process. This model was based on the application of the macroscopic mass conservation law, the Population Balance Equation (PBE) and the assumptions of complete mixing and isothermal conditions. The PBE is the most suitable approach to build dynamic models of particulate processes since it accounts for the polydispersed nature of such processes. This model accounted for the main event taking place within the reactor:

*An early version of this article was presented at the SIMS 2001 conference, October 8th9th, Porsgrunn, Norway.

† Author to whom correspondence should be addressed: B. Lie, Telemark University College, Department of Automation, P.O. Box 203, N-3901, Porsgrunn, Norway

$\$$ Elkem Research Centre, P.O. Box 8040, Vaagsbygd, N-4675 Kristiansand, Norway

$\S$ Fjeld Consulting, Meltzers gt. 4, N-0257, Oslo, Norway 
the disintegration of particles, but it could not represent properly the practical operation of the reactor.

The model of the hydrometallurgical leaching reactor has been reviewed recently, and extended to resemble more closely the operation of the reactor. The main modification is the inclusion of the condition governing the entrainment of particles in the outflow, thus permitting a distinction between the Particle Size Distribution (PSD) of the outflow and the PSD within the reactor, as it happens in reality. Another major change is the formulation of the energy balance that was necessary since temperature has an important influence on the operation of the reactor.

The resulting mathematical model is highly nonlinear and is composed of one partial integro-differential equation, ordinary differential equations and algebraic equations. Such a system is difficult to solve. The solution of the model is approached by discretizing the PBE in the particle size space, which reformulates the system into a system of Differential and Algebraic Equations (DAE). Historically, sets of DAEs were frequently reformulated and solved as Ordinary Differential Equations (ODE), by differentiation and/or extensive algebraic manipulation. However, it is advantageous to keep the system in the DAE form, mainly because the variables have a physical significance that is lost when reformulating the DAEs to ODEs. However, DAEs are not ODEs and difficulties can be encountered when using numerical methods to solve DAEs (Petzold, 1985). DAEs are solvable by numerical methods provided they are low index and consistent initial conditions are given. Thus, DAEs require analysis and characterization and may require reformulation before approaching its numerical solution.

The paper is organized as follows: In section 2, we give a brief description of the operation of the reactor in the Silgrain ${ }^{\mathbb{B}}$ process. In section 3 the model is discussed in detail, section 4 shows the analysis of the DAE system which results from the modeling stage and suggests the method to be used for the solution of the system. Section 5 presents some simulation results, and finally the conclusions are presented in section 6 .

\section{Operation of the reactor}

Leaching is the dissolution of a soluble constituent from a solid by means of a solvent. Aas (1971) described the process of hydrometallurgical leaching of ferrosilicon in detail. Silicon metal is produced by leaching lumps of $90-94 \% \mathrm{FeSi}$ in a hot acidic solution of ferric chloride and hydrochloric acid. The acid dissolves the impurities, mainly $\mathrm{Fe}, \mathrm{Al}$ and $\mathrm{Ca}$, breaking apart the lumps and leading to a granulated product of high purity (Si). The dissolution of impurities is assumed to proceed according to reduction-oxidation equations (Aas, 1971; Margarido, Martins, Figueiredo and Bastos, 1993). One feature that characterizes the Silgrain ${ }^{\circledR}$ process and distinguishes it from other hydrometallurgical leaching processes is the rapid disintegration of FeSi into small grains during the reaction (Aas, 1971).

A simplified sketch of the reactor is shown in Figure 1. The hot acidic solution is continuously pumped into the bottom of the vertical reactor and it leaves the reactor through an overflow pipe near the top, together with the disintegrated product. FeSi lumps are fed on the top of the reactor and sink to the bottom. The contact of the lumps with the hot acid causes the disintegration of the lumps, and the smallest grains are displaced upwards by the acid upflow (Aas, 1971).

The high flowrate of acid and the gas evolved during leaching causes agitation 

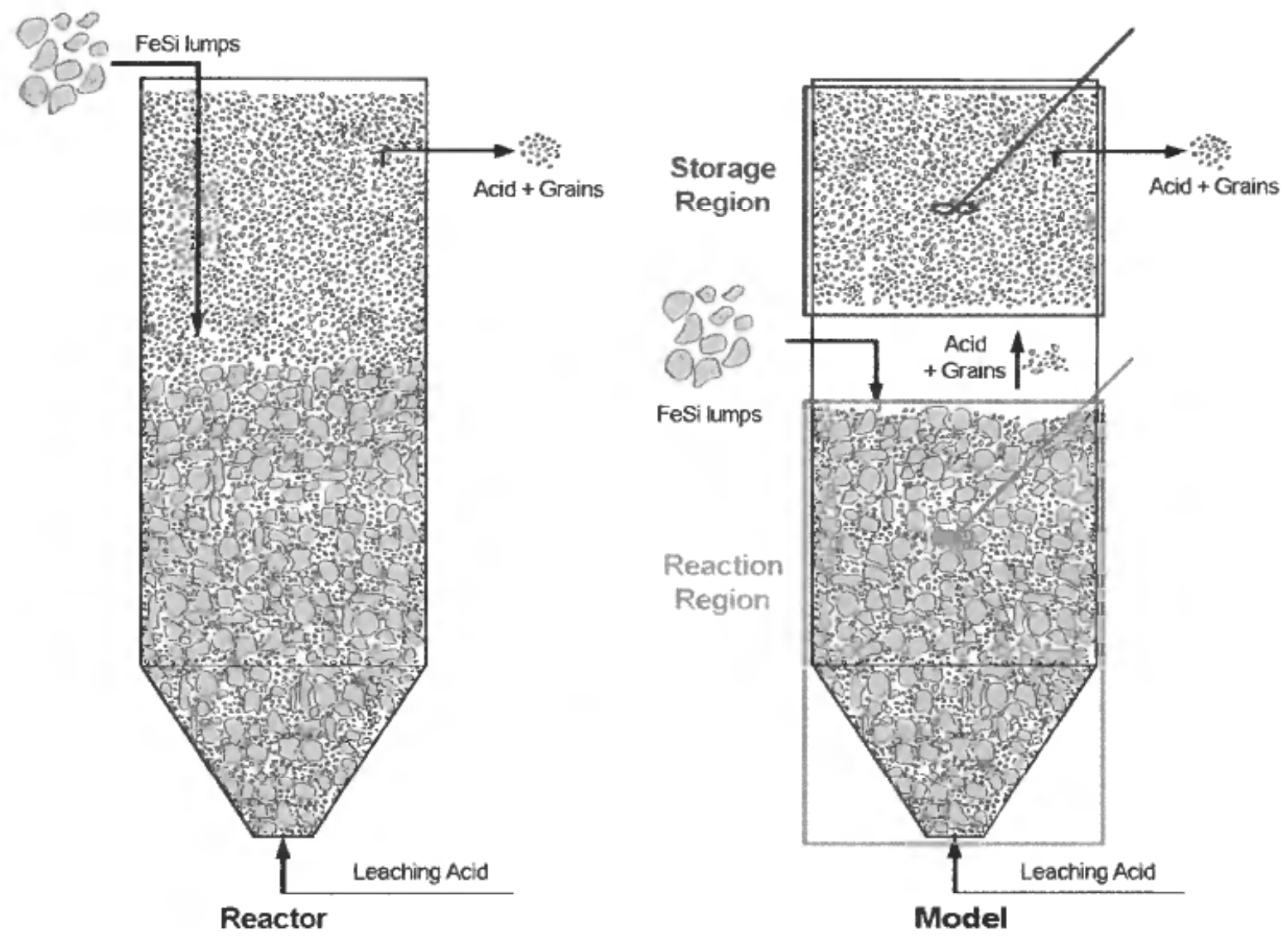

Figure 1. Sketch of reactor and model of the reactor.

within the reactor, but gravity classification of the solid charge still occurs. The upper layers consist of the fine particles that are displaced by the upward flow, whereas the deeper layers consist of unreacted and partially-reacted lumps of FeSi.

Clearly the assumption of complete mixing is not realistic, since only the disintegrated material that is small enough to be displaced by the acid upflow, flows out of the reactor. This means that the PSD at the outlet differs from the PSD within the reactor.

Aas (1971) stresses the importance of operating with FeSi lumps of the right composition, as well as using the right type of leach liquor. Temperature and leaching time play also an important role. This means that it is important to include the energy balance in the model.

\section{Model}

The reactor is modeled as one well-mixed reaction region and one well-mixed storage region in series. Figure 1 compares schematically the operation of the reactor and the model.

The residence time in the upper layers of the reactor, a mixture of acid and fine particles, is much shorter than the residence time in the bottom part. Therefore, it is reasonable to assume that no disintegration or reaction occur in the upper part of the reactor, so we can model it as a storage tank. Moreover, the FeSi lumps sink quickly through the upper layers, and do not accumulate there, so that it can be assumed that the feed enters directly into the well-mixed reaction region.

The high acid flowrate and the generation of gas during the reactions ensures 


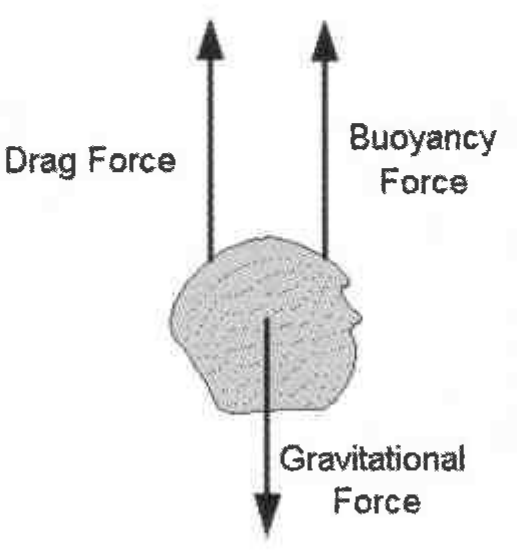

Figure 2. Forces acting on a particle.

agitation and well mixing conditions in both regions. Although we can assume homogeneity conditions within the reaction region, the PSD of particles leaving this region is not the same as the PSD of the particles remaining in that region. The free-falling velocity of the particles is the factor that determines whether the particles remain in the bottom part or flow up with the acid. As shown in Figure 2, each particle is subjected to three forces: the gravitational force, the drag force and the buoyancy force. For a certain particle diameter, to which we will refer as cut-size, the free-falling velocity equals the velocity of the upflow acid. Particles smaller than that cut size are displaced by the upflow, and particles larger than the cut size are not displaced.

The model suggested here consists in the application of the PBE to the reaction region and to the storage region, the mass conservation law to the reaction region and to the storage region and the energy balance to the whole reactor. These are discussed in more detail in the following sections.

\subsection{Population Balance Equation}

In 1964, two groups of researchers, Hulburt and Katz (1964) and Randolph (1964), observed that many problems involving change in particulate systems could not be handled within the framework of the conventional conservation equations. These researchers recognized that particulate materials are unique in that the dispersed phase is made up of a countable number of entities, and these entities possess a distribution of properties. They proposed the use of an equation for the continuity of particulate numbers, termed population balance, as a basis for describing the behavior of such systems. This number balance is developed from the general conservation equation

$$
\text { accumulation }=\text { input }- \text { output }+ \text { net generation }
$$

applied to particles having a specified set of properties. In the population balance, input and output terms represent changes in the number of particles in the specified property interval due to convective flow, while the net generation term accounts for particles entering and leaving the specified property intervals as a result of continuous processes such as chemical reaction, or discrete generation such as particle breakage.

The PBE has proven to be a powerful tool for quantifying the dynamics of many varied particle technologies such as crushing, agglomeration, liquid-liquid extraction, biochemical processes, emulsion polymerization, etc. Randolph and Larson (1988) 
and Ramkrishna (1985) present a complete review of the application of PBEs to problems in chemical engineering. This approach was first applied to hydrometallurgical leaching in single steady-state reactors by Herbst (1979). Rubisov and Papangelakis $(1995,1996 a, 1996 b)$ were the first to successfully model the transient behavior of hydrometallurgical leaching reactors.

In the field of reactive particulate processes, the macroscopic version of the PBE is commonly used

$$
\begin{aligned}
\frac{d \psi\left(D_{p}, t\right)}{d t}+\frac{\psi\left(D_{p}, t\right)}{V(t)} \frac{d V(t)}{d t}= & \frac{1}{V(t)}\left(Q_{\mathrm{in}}(t) \psi_{\mathrm{in}}\left(D_{p}, t\right)-Q(t) \psi\left(D_{p}, t\right)\right) \\
& -\frac{\partial}{\partial D_{p}}\left(\frac{d D_{p}}{d t} \psi\left(D_{p}, t\right)\right)+B-D
\end{aligned}
$$

where $\psi\left(D_{p}, t\right)\left(\mathrm{m}^{-4}\right)$ is the number-density PSD function per unit working volume and only one distributed property, the particle diameter $D_{p}$, is considered. The left hand side of equation (1) is the accumulation term, where $V(t)$ represents the working volume of the reactor $\left(\mathrm{m}^{3}\right)$, which is a function of time. The first two terms of the right hand side of equation (1) represent the input and output by convective transport, where $Q_{\mathrm{in}}(t)$ and $Q(t)$ are the slurry flow rate $\left(\mathrm{m}^{3} / \mathrm{s}\right)$ at the inlet and outlet, respectively. The generation term consists of two parts: a continuous disappearance of particles,

$$
\frac{\partial}{\partial D_{p}}\left(\frac{d D_{p}}{d t} \psi\left(D_{p}, t\right)\right)
$$

and a discrete generation of particles represented by the birth and death of particles by disintegration (the last two terms of the right hand side).

In the reaction region of the Silgrain reactor the two main events taking place are: disintegration of the particles and convective transport, and therefore, the PBE reduces to:

$$
\frac{d \psi_{1}\left(D_{p}, t\right)}{d t}+\frac{\psi_{1}\left(D_{p}, t\right)}{V_{1}(t)} \frac{d V_{1}(t)}{d t}=\frac{1}{V_{1}(t)}\left(\dot{M}_{\mathrm{in}}(t) f_{\mathrm{in}}\left(D_{p}, t\right)-q_{\mathrm{inter}}(t) \psi_{\mathrm{inter}}\left(D_{p}, t\right)\right)+B-D
$$

where $\dot{M}_{\text {in }}(t)$ is the mass input of FeSi $(\mathrm{kg} / \mathrm{s}) f_{\text {in }}\left(D_{p}, t\right)$ is the PSD of the FeSi expressed as weight-based probability distribution function $\left(\mathrm{m}^{-1}\right), V_{1}(t)$ is the volume of the reaction region, $\psi_{1}\left(D_{p}, t\right)$ is the PSD function per unit working volume within the reaction region, $\psi_{\text {inter }}\left(D_{p}, t\right)$ is the PSD leaving the reaction region and entering the storage region, $B$ represents the birth of particles of size $D_{p}$ resulting from breakage of larger particles and the $D$ represents the disappearance of particles of size $D_{p}$ due to disintegration. Let us discuss some of the terms in more detail.

1. The relationship between $\psi_{1}\left(D_{p}, t\right)$ and $\psi_{\text {inter }}\left(D_{p}, t\right)$ is given by the balance of forces acting on the particles. As discussed earlier, particles larger than the cut size remain in the reaction region and particles smaller than the cut size are displaced by the upflowing acid. Assuming that fines are displaced in the same concentration as within the reactor, the relationship of the two PSDs can be written as follows:

$$
\psi_{\text {inter }}\left(D_{p}, t\right)=\left\{\begin{array}{cc}
\psi_{1}\left(D_{p}, t\right) & \text { for } D_{p} \leqslant D_{\text {cut }} \\
0 & \text { for } D_{p}>D_{\text {cut }}
\end{array}\right.
$$


The cut size can be obtained from the balance of forces on the particle

$$
\text { Drag Force }=\text { Gravity Force }- \text { Buoyancy Force }
$$

and if we assume the particles are spherical (Coulson and Richardson, 1978)

$$
R_{o} \frac{\pi}{4} D_{\text {cut }}^{2}=\frac{\pi}{6} D_{\text {cut }}^{3} \rho_{p} g_{r}-\frac{\pi}{6} D_{\text {cut }}^{3} \rho_{f} g_{r}
$$

where $R_{o}$ is the dimensionless drag force, $\rho_{p}$ and $\rho_{f}$ are the solid density and the fluid density, respectively, and $g_{r}$ is the gravity constant. The drag force is generally a complicated function of the Reynolds number Re, but for turbulent flow $\left(500<\operatorname{Re}<2 \cdot 10^{5}\right)$, as in the reactor under study, the drag force is independent of the Reynolds number (Coulson and Richardson, 1978)

$$
\frac{R_{o}}{\rho_{f} u_{0}^{2}}=0.22
$$

where $u_{0}$ is the fluid velocity. Substituting equation (5) into equation (4) gives

$$
\begin{aligned}
D_{\text {cut }} & =0.33 \frac{\rho_{f}}{g_{r}\left(\rho_{p}-\rho_{f}\right)} u_{0}^{2} \\
& =0.33 \frac{\rho_{f}}{g_{r}\left(\rho_{p}-\rho_{f}\right)}\left(\frac{u_{e t}}{\left(1-g_{2}\right)^{n}}\right)^{2}
\end{aligned}
$$

where the fluid velocity has been expressed as a function of the the superficial fluid velocity $u_{e t}$ and the porosity $\left(1-g_{2}\right)$ in the storage region.

2. The discrete generation of particles in a certain particle diameter range is the result of the disintegration process. Such an event is typically represented by the particle birth and particle death rates, $B$ and $D$, respectively. No references have been found of the application of the PBE to leaching processes where the particles experienced disintegration. However, numerous references have been found of the application of PBE that include the birth and death rate functions in other fields of particulate processing such as granulation (Kapur, 1995), comminution (Herbst and Asihene, 1993; Ramkrishna, 1985), and emulsion dispersion (Chen, Prüss and Warbecke, 1998). It is important to note that although these particulate processes differ considerably, they model the birth and death rate terms in an identical way, as follows:

$$
B-D=\int_{D_{p^{\prime}}}^{\infty} b\left(D_{p}, y\right) a(y) \eta\left(D_{p}, y\right) \psi_{1}(y, t) d y-a\left(D_{p}\right) \psi_{1}\left(D_{p}, t\right)
$$

where $a\left(D_{p}\right)$ is the particle breakup frequency function $\left(\mathrm{s}^{-1}\right), b\left(D_{p}, y\right)$ is the probability distribution function of daughter particles of size $D_{p}$ by breakage of a particle of size $y$, and $\eta\left(D_{p}, y\right)$ is the average number of daughter particles by breakage of one particle of size $y$. Such a mathematical formulation for the discrete generation is used here. Batch laboratory experiments have been carried out to determine expressions for the particle breakup frequency function and the probability distribution function. The particle breakup frequency function depends mainly on the particle size and temperature and when particles are as small as the average grain size they do not disintegrate anymore. We thus propose the following expression 


$$
a\left(D_{p}, T\right)=\left\{\begin{array}{cl}
\frac{k_{a} \exp \left(k_{T} T\right)}{D_{p}^{n}} & \text { for } D_{p} \geqslant D_{\text {grain }} \\
0 & \text { for } D_{p}<D_{\text {grain }}
\end{array}\right.
$$

for the particle breakup frequency function, where $k_{a}, k_{T}$ and $n$ are the fitting parameters and $D_{\text {grain }}$ depends on the quality of the feed material used in the process. Regarding the probability distribution function of daughter particles, the laboratory results showed a bimodal distribution function, in which one of the modes is static at a certain particle size diameter $D_{p}^{*}$ and the other one depends on the size of the mother particle $y$. The distribution functions commonly encountered in the references did not fit the data well. We suggest then the following expression:

$$
b\left(D_{p}, y\right)=A_{1} \exp \left(-\beta_{1}\left(1-\frac{D_{p}}{D_{p}^{*}}\right)^{2}\right)+\frac{A_{2}}{D_{p}} \exp \left(-\beta_{2} \log ^{2}\left(1-\frac{D_{p} y}{\left(y-D_{p}\right) D_{p}^{*}}\right)\right)
$$

which shows good fitting properties to the experimental data, and where $A_{1}$, $\beta_{1}, A_{2}, \beta_{2}$ and $D_{p}^{*}$ are the fitting parameters. Finally, the average number of daughter particles $\eta\left(D_{p}, y\right)$ by breakage of one particle of size $y$ can be obtained from the dimensional analysis of equation (8). The suggested expressions have the following dimensions: $b\left(D_{p}, y\right)$ gives the mass of particles of size $D_{p}$ obtained per unit mass of mother particle of size $y$ that experiences breakage, $\psi_{1}(y, t)$ gives the number of mother particles of size $y$ per unit working volume, $a(y, T)$ gives the rate of breakup of a mother particle of size $y$ and $B$ is the number of particles of size $D_{p}$ that are created per unit time and per unit working volume. Therefore, in order to match dimensions, $\eta\left(D_{p}, y\right)$ is the ratio between the mass of one mother particle and the mass of one daughter particle

$$
\eta\left(D_{p}, y\right)=\frac{\rho_{p} \pi / 6 y^{3}}{\rho_{p} \pi / 6 D_{p}^{3}}=\frac{y^{3}}{D_{p}^{3}}
$$

In the storage region of the Silgrain reactor the main event taking place is the convective transport, and therefore, the PBE reduces to:

$$
\frac{d \psi_{2}\left(D_{p}, t\right)}{d t}+\frac{\psi_{2}\left(D_{p}, t\right)}{V_{2}(t)} \frac{d V_{2}(t)}{d t}=\frac{1}{V_{2}(t)}\left(q_{\text {inter }}(t) \psi_{\text {inter }}\left(D_{p}, t\right)-q_{\text {out }}(t) \psi_{2}\left(D_{p}, t\right)\right)
$$

where the inlet flow is equal to the outlet flow from the reaction region and where the PSD within the storage region is the same as the PSD in the outlet flow since the assumption of complete mixing is appropriate.

\subsection{Mass Balance}

The macroscopic mass conservation law applied to the reaction part of the reactor can be written as follows

$$
\begin{aligned}
\frac{d M_{1}(t)}{d t} & =\dot{M}_{\text {in }}+q_{\text {in }} \rho_{\text {in }}-\frac{q_{\text {in }}}{1-g_{\text {inter }}} \rho_{\text {inter }}(t) \\
\frac{d}{d t}\left(V_{1}(t) \rho_{1}(t)\right. & =\dot{M}_{\text {in }}+q_{\text {in }} \rho_{\text {in }}-\frac{q_{\text {in }}}{1-g_{\text {inter }}} \rho_{\text {inter }}(t)
\end{aligned}
$$


where $\dot{M}_{\text {in }}$ is the mass input of FeSi, $q_{\text {in }}$ is the volumetric inflow of leaching acid and $\rho_{1}(t)$ and $\rho_{\text {inter }}(t)$ are the slurry densities within the reaction region and in the outlet flow from the reaction region, respectively. Densities are a function of the solid's volume fraction, as given by

$$
\begin{gathered}
\rho_{1}(t)=\rho_{f}\left(1-g_{1}(t)\right)+\rho_{p} g_{1}(t) \\
\rho_{\text {inter }}(t)=\rho_{f}\left(1-g_{\text {inter }}(t)\right)+\rho_{p} g_{\text {inter }}(t)
\end{gathered}
$$

In turn, the solid's volume fraction is a function of the third moment of the PSD

$$
\begin{aligned}
g_{1}(t) & =\frac{\pi}{6} \int_{0}^{D_{p \max }} D_{p}^{3} \psi_{1}\left(D_{p}, t\right) d D_{p} \\
g_{\text {inter }}(t) & =\frac{\pi}{6} \int_{0}^{D_{p \max }} D_{p}^{3} \psi_{\text {inter }}\left(D_{p}, t\right) d D_{p}
\end{aligned}
$$

It must be noted that equation (12) assumes that the acid does not accumulate in the reaction region and that the outflow is thus composed of the acid inflow plus the fines displaced by the acid. This assumption is reasonable according to the operation of the industrial reactor, since the acid is fed continuously.

Now, the application of the macroscopic mass conservation law to the storage region of the reactor results in the following expressions

$$
\begin{aligned}
\frac{d M_{2}(t)}{d t} & =\frac{q_{\text {in }}}{1-g_{\text {inter }}} \rho_{\text {inter }}(t)-q_{\text {out }}(t) \rho_{2}(t) \\
\frac{d}{d t}\left(V_{2}(t) \rho_{2}(t)\right) & =\frac{q_{\text {in }}}{1-g_{\text {inter }}} \rho_{\text {inter }}(t)-q_{\text {out }}(t) \rho_{2}(t)
\end{aligned}
$$

where

$$
\begin{aligned}
& \rho_{2}(t)=\rho_{f}\left(1-g_{2}(t)\right)+\rho_{p} g_{2}(t) \\
& g_{2}(t)=\frac{\pi}{6} \int_{0}^{D_{p \max }} D_{p}^{3} \psi_{2}\left(D_{p}, t\right) d D_{p}
\end{aligned}
$$

and $q_{\text {out }}(t)$ occurs by gravity and can be derived by applying Bernoulli's law between the surface of the liquid and the physical outlet, yielding to

$$
q_{\text {out }}=C_{D} \frac{\pi}{4} D_{\text {out }}^{2} \sqrt{2 g_{r}\left(\frac{4}{\pi D_{r}^{2}}\left(V_{1}+V_{2}-V_{\text {semiconical }}\right)-h_{\text {out }}\right)}
$$

where $D_{r}$ is the diameter of the cylindrical part of the reactor, $h_{\mathrm{out}}$ is the distance between the bottom of the cylindrical part of the reactor and the outlet orifice, $V_{\text {semiconical }}$ is the volume of the reactor that has semiconical shape, $D_{\text {out }}$ is the diameter of the outlet orifice, and $C_{D}$ is the coefficient of discharge, which typically has a value of 0.64 for liquids or slurries (Coulson and Richardson, 1978).

\subsection{Energy Balance}

Temperature has an important influence on the disintegration of particles. This makes it necessary to use a nonisothermal model. In the model suggested here, we 
assume that the temperature is the same both in the reaction region and in the storage region of the reactor. Such an assumption is reasonable since the main heat input is provided by the acid inflow, and the corresponding flowrate is very high in comparison to the FeSi feed, causing great turbulence within the reactor and therefore good heat transmission, with the FeSi feed being heated to the reactor temperature almost instantly.

Therefore, the energy balance applied to the whole reactor is given by

$$
\frac{d U(t)}{d t}=h_{\text {in }}-h_{\text {out }}-Q
$$

where $U(t)$ is the internal energy of the system, $h_{\text {in }}$ and $h_{\text {out }}$ are the enthalpy flow entering and leaving the reactor, respectively and $Q$ is the heat loss to the surroundings. It must be noted that in the energy balance, the kinetic and the gravitational components of energy have been neglected in comparison to the internal energy component $U(t)$. Moreover, we assume that the internal energy and the enthalpies are functions of temperature, so that we can rewrite equation (21) as follows

$$
\begin{aligned}
& \frac{d}{d t}\left\{V_{1}(t) \rho C p_{1}(t)\left(T(t)-T_{\text {ref }}\right)+V_{2}(t) \rho C p_{2}(t)\left(T(t)-T_{\text {ref }}\right)\right\} \\
& =\dot{M}_{\text {in }}(t) C p_{p}\left(T_{s}-T_{\text {ref }}\right)+q_{\text {in }} \rho_{f} C p_{f}\left(T_{\text {in }}-T_{\text {ref }}\right)-q_{\text {out }}(t) \rho C p_{2}(t)\left(T(t)-T_{\text {ref }}\right)
\end{aligned}
$$

where $C p_{p}$ and $C p_{l}$ are the heat capacities $(\mathrm{kcal} / \mathrm{kg} \mathrm{K})$ of $\mathrm{FeSi}$ and the acid, respectively, $T_{s}$ is the surroundings temperature (K), $T_{\text {ref }}$ is the reference temperature $(\mathrm{K})$ used to calculate enthalpies, $\rho C p_{1}(t)$ and $\rho C p_{2}(t)$ are the average heat capacity $\left(\mathrm{kcal} / \mathrm{m}^{3} \mathrm{~K}\right)$ of the slurry within the reaction region and within the storage region, respectively. The average heat capacities are functions of the solid's volume fraction as shown below

$$
\begin{aligned}
& \rho C p_{1}(t)=\rho_{f} C p_{f}\left(1-g_{1}(t)\right)+\rho_{p} C p_{p} g_{1}(t) \\
& \rho C p_{2}(t)=\rho_{f} C p_{f}\left(1-g_{2}(t)\right)+\rho_{p} C p_{p} g_{2}(t)
\end{aligned}
$$

\subsection{Overview of the model}

Table 1 summarizes the model, which consists of 1 integrodifferential equation, 4 implicit ordinary differential equations, 8 algebraic equations and 3 integral equations.

\subsection{Discretization of the model}

Integrodifferential equations are very difficult to solve. The method of moments, as developed by Hulburt and Katz (1964), is the most widely used method to reduce integrodifferential equations into a set of ordinary equations, but it is not applicable here because this would lead to an open set of equations.

Examples of numerical solution methods proposed in the literature (Ramkrishna, 1985) are: the method of weighted residuals, the method of self-preserving distributions, Monte Carlo simulation techniques, the size interval-by-size marching method and discretization via fixed/moving pivot techniques. Of the numerical methods, those based on discretization of the continuous PBE are reported to be the most attractive from the computational point of view (Kumar and Ramkrishna, 1996).

Discretization techniques aim at the formulation of PBE in discrete particle size 
Table 1. Overview of the model

$$
\begin{aligned}
\frac{d \psi_{1}\left(D_{p}, t\right)}{d t}+\frac{\psi_{1}\left(D_{p}, t\right)}{V_{1}(t)} \frac{d V_{1}(t)}{d t}= & \frac{1}{V_{1}(t)}\left(\dot{M}_{\mathrm{in}}(t) f_{\mathrm{in}}\left(D_{p}, t\right)-\frac{q_{\mathrm{in}}}{1-g_{\mathrm{inter}}(t)} \psi_{\mathrm{inter}}\left(D_{p}, t\right)\right) \\
& +\int_{D_{p}}^{\infty} b\left(D_{p}, y\right) \eta\left(D_{p}, y\right) a\left(D_{p}, T\right) \psi_{1}(y, t) d y-a\left(D_{p}, T\right) \psi_{1}\left(D_{p}, t\right)
\end{aligned}
$$

where $\quad a\left(D_{p}, T\right)=\frac{k_{a} \exp \left(k_{T} T\right)}{D_{p}^{n}}$

and $\quad b \eta\left(D_{p}, y\right)=A_{1} \exp \left(-\beta_{1}\left(1-\frac{D_{p}}{D_{p}^{*}}\right)^{2}\right)+\frac{A_{2}}{D_{p}} \exp \left(-\beta_{2} \log ^{2}\left(1-\frac{D_{p} y}{\left(y-D_{p}\right) D_{p}^{*}}\right)\right) \frac{y^{3}}{D_{p}^{3}}$

$\psi_{\text {inter }}\left(D_{p}, t\right)=\left\{\begin{array}{cc}\psi_{1}\left(D_{p}, t\right) & \text { for } D_{p} \leqslant D_{\text {cut }} \\ 0 & \text { for } D_{p}>D_{\text {cut }}\end{array}\right.$

$D_{\text {cut }}=0.33 \frac{\rho_{f}}{g_{r}\left(\rho_{p}-\rho_{f}\right)}\left(\frac{u_{e t}}{\left(1-g_{2}(t)\right)^{n}}\right)^{2}$

$\frac{d}{d t}\left(V_{1}(t) \rho_{1}(t)\right)=\dot{M}_{\text {in }}+q_{\text {in }} \rho_{\text {in }}-\frac{q_{\text {in }}}{1-g_{\text {inter }}(t)} \rho_{\text {inter }}(t)$

$\rho_{1}(t)=\rho_{f}\left(1-g_{1}(t)\right)+\rho_{p} g_{1}(t)$

$\rho_{\text {inter }}(t)=\rho_{f}\left(1-g_{\text {inter }}(t)\right)+\rho_{p} g_{\text {inter }}(t)$

$g_{1}(t)=\frac{\pi}{6} \int_{0}^{D_{p \max }} D_{p}^{3} \psi_{1}\left(D_{p}, t\right) d D_{p}$

$g_{\text {inter }}(t)=\frac{\pi}{6} \int_{0}^{D_{p \max }} D_{p}^{3} \psi_{\text {inter }}\left(D_{p}, t\right) d D_{p}$

$\frac{d \psi_{2}\left(D_{p}, t\right)}{d t}+\frac{\psi_{2}\left(D_{p}, t\right)}{V_{2}(t)} \frac{d V_{2}(t)}{d t}=\frac{1}{V_{2}(t)}\left(\frac{q_{\text {in }}}{1-g_{\text {inter }}(t)} \psi_{\text {inter }}\left(D_{p}, t\right)-q_{\text {out }}(t) \psi_{2}\left(D_{p}, t\right)\right)$

$q_{\text {out }}(t)=C_{D} \frac{\pi}{4} D_{\text {out }}^{2} \sqrt{2 g_{r}\left(\frac{4}{\pi D_{r}^{2}}\left(V_{1}(t)+V_{2}(t)-V_{\text {semiconical }}\right)-h_{\text {out }}\right)}$

$\frac{d}{d t}\left(V_{2}(t) \rho_{2}(t)\right)=\frac{q_{\text {in }}}{1-g_{\text {inter }}} \rho_{\text {inter }}(t)-q_{\text {out }}(t) \rho_{2}(t)$

$p_{2}(t)=\rho_{f}\left(1-g_{2}(t)\right)+\rho_{p} g_{2}(t)$

$g_{2}(t)=\frac{\pi}{6} \int_{0}^{D_{p \max }} D_{p}^{3} \psi_{2}\left(D_{p}, t\right) d D_{p}$

$\frac{d}{d t}\left\{V_{1}(t) \rho C p_{1}(t)\left(T(t)-T_{\mathrm{ref}}\right)+V_{2}(t) \rho C p_{2}(t)\left(T(t)-T_{\mathrm{ref}}\right)\right\}$

$=\dot{M}_{\text {in }} C p_{p}\left(T_{s}-T_{\text {ref }}\right)+q_{\text {in }} \rho_{f} C p_{f}\left(T_{\text {in }}-T_{\text {ref }}\right)-q_{\text {out }}(t) \rho C p_{2}(t)\left(T(t)-T_{\text {ref }}\right)-Q$

$\rho C p_{1}(t)=\rho_{f} C p_{f}\left(1-g_{1}(t)\right)+\rho_{p} C p_{p} g_{1}(t)$

$\rho C p_{2}(t)=\rho_{f} C p_{f}\left(1-g_{2}(t)\right)+\rho_{p} C p_{p} g_{2}(t)$ 
space. This is done by integrating the continuous PBE over a discrete size interval, say $D_{p, i}$ to $D_{p, i+1}$,

$$
\begin{aligned}
\int_{D_{p, i}}^{D_{p, i+1}} \frac{1}{V} \frac{\partial(V \psi)}{\partial t} d D_{p}= & \int_{D_{p, i}}^{D_{p, i+1}} \frac{1}{V}\left(Q_{\text {in }} \psi_{\text {in }}-Q \psi\right) d D_{p}-\int_{D_{p, i}}^{D_{p, i+1}} a\left(D_{p}\right) \psi\left(D_{p}, t\right) d D_{p} \\
& +\int_{D_{p, i}}^{D_{p, i+1}} \int_{D_{p}}^{D_{p, \max }} b\left(D_{p}, y\right) a(y) \eta\left(D_{p}, y\right) \psi(y, t) d y d D_{p}
\end{aligned}
$$

where the discrete PSD, $\phi_{i}(t)$ is given by

$$
\phi_{i}(t)=\int_{D_{p, i}}^{D_{p, i+1}} \psi\left(D_{p}, t\right) d D_{p}
$$

and the number of intervals used to represent the total population of particles is $N$. Thus, $i=1,2, \ldots N$. The various discretization methods differ in the way of relating the continuous and discrete PSDs, $\psi\left(D_{p}, t\right)$ and $\phi_{i}(t)$.

The main disadvantage of discretization methods is that the discretized model may not be consistent with the number and mass conservation laws, or any other integral property of interest associated to the entire population. Often, the accuracy of the solutions is improved by using finer discretization grids, but this is incurring a very high computational costs. The method proposed by Kumar and Ramkrishna (1996) improves considerably the effectiveness of discretization. They suggest that the discrete equations are internally consistent with regard to the desired moments of interest of the distribution, thus ensuring the preservation of the quantities of interest, while relaxing the accuracy of other less important quantities.

The numerical technique divides the entire size range into small sections. The size range contained in between two sizes $D_{p, i}$ and $D_{p, i+1}$ is called the $i$ th section. The particle population in this range is represented by a size $x_{i}$, such that $D_{p, i}<x_{i}<D_{p, i+1}$. This technique allows for the use of a general grid, for example, fine in some ranges and coarse elsewhere. The particle population is assumed to be concentrated at representative sizes, $x_{i}$ 's, having a zero value for other sizes. Hence, the continuous and the discrete PSDs, $\psi\left(D_{p}, t\right)$ and $\phi_{i}(t)$, are related as follows

$$
\psi\left(D_{p}, t\right)=\sum_{i=1}^{N} \phi_{i}(t) \dot{\delta}\left(D_{p}-x_{i}\right)
$$

where $\delta\left(D_{p}-x_{i}\right)$ is the Delta Dirac function. Once this method is applied to the system under study we obtain the discretized model shown in Table 2 . The discretized system of equations is thus an implicit nonlinear DAE system.

\section{Analysis of the DAE}

Historically, sets of DAEs were frequently restated and solved as ODEs, by differentiation and/or extensive algebraic manipulation, often destroying the natural structure of the system. Today, it is becoming more common to deal with such problems in their original, natural DAE form, mainly because the variables in the original DAE typically have some physical significance, whereas those that result after manipulation into an ODE may not (Lefkopoulos and Stadherr, 1993). However, 
Table 2. Discretized model

$\frac{d \phi_{1, i}(t)}{d t}+\frac{\phi_{1, i}(t)}{V_{1}(t)} \frac{d V_{1}(t)}{d t}=\frac{1}{V_{1}(t)}\left(\dot{M}_{\text {in }}(t) F_{\text {in }}(t)-\frac{q_{\text {in }}}{1-g_{\text {inter }}(t)} \phi_{\text {inter }, i}(t)\right)+\sum_{k>i}^{N} n_{i, k} a_{k} \phi_{k}(t)-a_{i} \phi_{i}(t)$

where $\quad i=1,2, \ldots N$,

$$
\begin{aligned}
n_{i, k} & =\int_{x_{i}}^{x_{i+1}}\left(\frac{x_{i+1}^{3}-D_{p}^{3}}{x_{i+1}^{3}-x_{i}^{3}}\right) b \eta\left(D_{p}, x_{k}\right) d D_{p}+\int_{x_{i-1}}^{x_{i}}\left(\frac{D_{p}^{3}-x_{i-1}^{3}}{x_{i}^{3}-x_{i-1}^{3}}\right) b\left(D_{p}, x_{k}\right) d D_{p}, \\
b \eta\left(D_{p}, x_{k}\right) & =\left(A_{1} \exp \left(-\beta_{1}\left(1-\frac{D_{p}}{D_{p}^{*}}\right)^{2}\right)+\frac{A_{2}}{D_{p}} \exp \left(-\beta_{2} \log ^{2}\left(1-\frac{D_{p} x_{k}}{\left(y-D_{p}\right) D_{p}^{*}}\right)\right)\right) \frac{y^{3}}{D_{p}^{3}} \\
\text { and } \quad a_{k} & =\frac{k_{a} \exp \left(k_{T} T\right)}{x_{k}^{n}}
\end{aligned}
$$$$
V_{1}(t) \frac{\left.d \rho_{1}(t)\right)^{\prime}}{d t}+p_{1}(t) \frac{d V_{1}(t)}{d t}=\dot{M}_{\text {in }}+q_{\text {in }} \rho_{\text {in }}-\frac{q_{\text {in }}}{1-g_{\text {inter }}(t)} \rho_{\text {inter }}
$$$$
g_{1}(t)=\frac{\pi}{6} \sum_{i=1}^{N} x_{i}^{3} \phi_{1, i}(t)
$$$$
\rho_{1}(t)=\rho_{f}\left(1-g_{1}(t)\right)+\rho_{p} g_{1}(t)
$$$$
D_{\text {cut }}=0.33 \frac{\rho_{f}}{g_{r}\left(\rho_{p}-\rho_{f}\right)}\left(\frac{u_{e t}}{\left(1-g_{2}(t)\right)^{n}}\right)^{2}
$$$$
\phi_{\text {inter }, i}(t)=\left\{\begin{array}{cc}
\phi_{1, i}(t) & \text { for } D_{p} \leqslant D_{\text {cut }} \\
0 & \text { for } D_{p}>D_{\text {cut }}
\end{array}\right.
$$$$
g_{\text {inter }}(t)=\frac{\pi}{6} \sum_{i=1}^{N} x_{i}^{3} \phi_{\text {inter }, i}(t)
$$$$
\rho_{\text {inter }}(t)=\rho_{f}\left(1-g_{\text {inter }}(t)\right)+\rho_{p} g_{\text {inter }}(t)
$$$$
\frac{d \phi_{2, i}(t)}{d t}+\frac{\phi_{2, i}(t)}{V_{2}(t)} \frac{d V_{2}(t)}{d t}=\frac{1}{V_{2}(t)}\left(\frac{q_{\text {in }}}{1-g_{\text {inter }}(t)} \phi_{\text {inter }, i}(t)-q_{\text {out }}(t) \phi_{2, i}(t)\right)
$$$$
V_{2}(t) \frac{d \rho_{2}(t)}{d t}+\rho_{2}(t) \frac{d V_{2}(t)}{d t}=\frac{q_{\mathrm{in}}}{1-g_{\mathrm{inter}}(t)} \rho_{\mathrm{inter}}(t)-q_{\mathrm{out}}(t) \rho_{2}(t)
$$$$
q_{\text {out }}(t)=C_{D} \frac{\pi}{4} D_{\text {out }}^{2} \sqrt{2 g\left(\frac{4}{\pi D_{r}^{2}}\left(V_{1}(t)+V_{2}(t)-V_{\text {semiconical }}\right)-h_{\text {out }}\right)}
$$$$
g_{2}(t)=\frac{\pi}{6} \sum_{i=1}^{N} x_{i}^{3} \phi_{2, i}(t)
$$

$$
\begin{aligned}
& p_{2}(t)=\rho_{f}\left(1-g_{2}(t)\right)+\rho_{p} g_{2}(t) \\
& \frac{d}{d t}\left\{V_{1}(t) \rho C p_{1}(t)\left(T(t)-T_{\mathrm{ref}}\right)+V_{2}(t) \rho C p_{2}(t)\left(T(t)-T_{\mathrm{ref}}\right)\right\} \\
& =\dot{M}_{\text {in }} C p_{p}\left(T_{s}-T_{\text {ref }}\right)+q_{\text {in }} \rho_{f} C p_{f}\left(T_{\text {in }}-T_{\text {ref }}\right)-q_{\text {out }}(t) \rho C p_{2}(t)\left(T(t)-T_{\text {ref }}\right)-Q \\
& \rho C p_{1}(t)=\rho_{f} C p_{f}\left(1-g_{1}(t)\right)+\rho_{p} C p_{p} g_{1}(t) \\
& \rho C p_{2}(t)=\rho_{f} C p_{f}\left(1-g_{2}(t)\right)+\rho_{p} C p_{p} g_{2}(t)
\end{aligned}
$$


differential and algebraic equations (DAEs) are not ordinary differential equations (ODEs) (Petzold, 1985). A number of difficulties can arise when numerical methods are used to solve DAE systems of the form $\mathbf{F}\left(t, \mathbf{y}, \mathbf{y}^{\prime}\right)=0$. Many of the DAE systems can be solved using numerical methods which are commonly used for solving stiff systems of ODEs, such as Backward Differentiation Formulas (BDF). Others can be solved using such methods but only after substantial modification to the strategies usually used in codes implementing those methods (Petzold, 1985).

DAE systems can be characterized using the concept of index. The index can be thought of as a measure of the variation of the DAE structure from a standard ODE system (Lefkopoulos and Stadherr, 1993). Therefore, the first thing to do when dealing with DAE systems is to determine its index. Let us define in more detail the concept of index of a DAE. It can be defined as the minimum number of times we must differentiate all or part of $\mathbf{F}\left(t, \mathbf{y}, \mathbf{y}^{\prime}\right)=0$ with respect to time $t$ in order to determine $\mathbf{y}^{\prime}$ as a continuous function of $\mathbf{y}$ and $t$. More formally, it can be stated that the index of a DAE is the smallest nonnegative integer $v$ such that $\mathbf{F}$ has $v$ continuous derivatives and the nonlinear system:

$$
\begin{aligned}
\mathbf{F}\left(t, \mathbf{y}, \mathbf{y}^{\prime}\right) & =0 \\
\frac{d}{d t} \mathbf{F}\left(t, \mathbf{y}, \mathbf{y}^{\prime}, \mathbf{y}^{\prime \prime}\right) & =\frac{d \mathbf{F}}{d \mathbf{y}} \mathbf{y}^{\prime}+\frac{d \mathbf{F}}{d \mathbf{y}^{\prime}} \mathbf{y}^{\prime \prime}+\frac{\partial \mathbf{F}}{\partial t}=0 \\
& \vdots \\
\frac{d^{v}}{d t^{v}} \mathbf{F}\left(t, \mathbf{y}, \mathbf{y}^{\prime}, \mathbf{y}^{\prime \prime}, \ldots, \mathbf{y}^{v}, \mathbf{y}^{v+1}\right) & =0
\end{aligned}
$$

when viewed as relating $t, \mathbf{y}, \mathbf{y}^{\prime}, \mathbf{y}^{\prime \prime}, \ldots, \mathbf{y}^{v}, \mathbf{y}^{v+1}$ as independent variables, is solved for $\mathbf{y}^{\prime}$ uniquely in terms of $\mathbf{y}$ and $t$, i.e. there is an underlying ODE $\mathbf{y}^{\prime}=\mathbf{y}^{\prime}(z, t)$. The definition implies that any pure ODE is an index-zero DAE, and a system of algebraic equations is index-one provided it is nonsingular.

For the purposes of index analysis of a DAE it is often convenient to partition the equations $\mathbf{F}$ into differential equations $\mathbf{f}$ and algebraic equations $\mathbf{g}$. Thus, without loss of generality, the DAE can be rewritten as follows

$$
\begin{aligned}
\mathbf{f}\left(t, \mathbf{y}, \mathbf{y}^{\prime}\right) & =0 \\
\mathbf{g}(t, \mathbf{y}) & =0
\end{aligned}
$$

It can be shown that the DAE is index-one if and only if the matrix

$$
B=\left(\begin{array}{l}
\frac{d \mathbf{f}}{d \mathbf{y}^{\prime}} \\
\frac{d \mathbf{g}}{d \mathbf{y}}
\end{array}\right)
$$

is nonsingular (Lefkopoulos and Stadherr, 1993).

If we apply the criteria in equation (28) to our DAE system, we obtain a matrix that is a function of $t$ and of the internal vector $\mathbf{y}$. Therefore, the index of the DAE system may vary during integration. It can be proved that the DAE under study is index-one as long as the volume of slurry within the reaction region and within the storage region is distinct from zero, or what is the same, the model is an index-one implicit DAE except when the reactor is empty (i.e., during start-up). The advantage of index-one systems is that they do not need reformulation in order to be solved. 
Another difference between ODEs and DAEs is that in the latter we must specify consistent initial conditions, and this can become a challenging problem for certain DAEs. For a set of initial conditions to be consistent, it must satisfy the system at an initial time $t_{0}$

$$
\mathbf{F}\left(t_{0}, \mathbf{y}_{0}, \mathbf{y}_{0}^{\prime}\right)=0
$$

Note that the term 'initial conditions' is used to refer to the vector $\left(\mathbf{y}_{0}, \mathbf{y}_{0}^{\prime}\right)$ rather than simply to $\mathbf{y}_{0}$. This is a necessary condition, but not always sufficient for consistency. Usually, some or all of the equations resulting from differentiating $\mathbf{F} v$ times with respect to time have to be satisfied too.

The most widely used approach to determination of consistent initial conditions is to reduce the system to an index-zero system. The system under study is index one, so in order to reduce the system to an index-zero system we have to differentiate the algebraic equations with respect to time once, as shown in Table 3.

Now, we can arbitrarily decide the initial value for the differential variables of the

Table 3. Derivatives of algebraic equations of the system

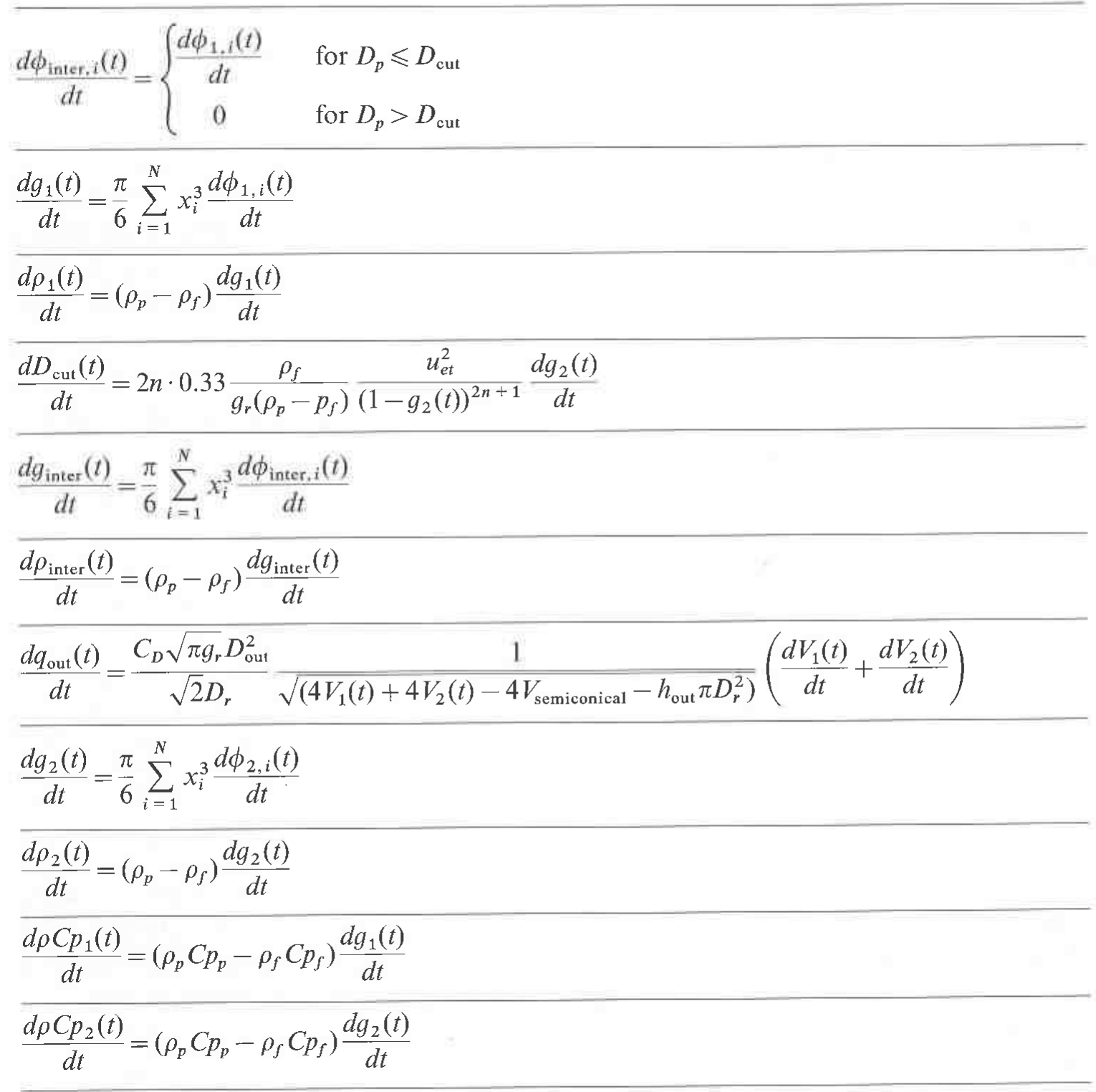


DAE, i.e. $\phi_{1, i}(t), V_{1}(t), \phi_{2, i}(t), V_{2}(t)$ and $T(t)$. Once this is done, the remaining initial conditions are calculated by solving the system of equations composed by the equations in Table 3 evaluated at $t=0$ and the differential equations in Table 2 evaluated at $t=0$. It must be noted that in the case under study some of the initial conditions can be calculated by direct substitution in the equations, but others result from the solution of a linear system of equations. By following this approach, we get a set of consistent initial conditions $\left(\mathbf{y}_{0}, \mathbf{y}_{0}^{\prime}\right)$.

It is useful to discuss in more detail the selection of the degrees of freedom. $V_{1}(0)$, $V_{2}(0)$ and $T(0)$ can be chosen making use of the knowledge of the operation of the reactor. However, to select the discrete number-density PSD functions per unit working volume, $\phi_{1, i}(t)$ and $\phi_{2, i}(t)$, is not straightforward. One possible approach, that is applied here, is the following:

1. Choose a continuous weight-density PSD.

2. Apply the discretization grid and integrate in order to calculate the discrete weight-density PSD

3. Transform the distribution from weight-basis to number-basis.

Initially, B-splines were used to fit a possible continuous weight-density PSD, but this approach was rejected because the interpolation between points could yield negative values for those regions of the distribution function close to zero and negative values do not have physical meaning in this context. Instead, it was decided to use a log-normal distribution function for the initial weight-based distribution function. The log-normal distribution is often used in the empirical representation of PSDs (Randolph and Larson, 1988). The variant used here can be used for distributions that are truncated below a minimum size and/or above a maximum size, and is written as follows

$$
f=\frac{1}{(2 \pi)^{0.5} \log \sigma} \exp \left\{-\log \left(\frac{\left(D_{p}-D_{p, \min }\right)\left(D_{p, \max }-D_{p, \min }\right)}{\left(D_{p, \max }-D_{p, \min }\right) \bar{D}_{p} 2^{0.5} \log \sigma}\right)^{2}\right\}
$$

where $D_{p, \min }$ is the minimum diameter, $D_{p, \max }$ is the maximum diameter, $\bar{D}_{p}$ is the average diameter and $\sigma$ is the standard deviation.

Finally, one issue that arose in this problem is the need of scaling the states and the system of equations. The variables of the system show a wide range. Hence, the discrete PSD can have a value in the order of $10^{9}$, whereas the cut-size diameter can have a value of the order of $10^{-3}$. Such a large difference in order of magnitude can cause ill-conditioning problems when calculating the Jacobian of the system of equations. In order to avoid such problems, the system of equations was rewritten in terms of scaled variables and the equations themselves were also scaled. In both cases, the scaling factor was the initial value of the variables except for those variables whose value is 0 .

Once the DAE has been categorized by its index, and consistent initial conditions have been calculated, we are in a condition to select the method of solution for the DAE system. Linear Multistep methods, and more precisely, the Backward Differentiation Formulas (BDF), have emerged as the most popular method to solve DAEs. The simplest first order BDF method is the implicit Euler method, which consists of replacing the derivative in $\mathbf{F}\left(t, \mathbf{y}, \mathbf{y}^{\prime}\right)=0$ by a backward difference

$$
\mathbf{F}\left(t_{n}, \mathbf{y}_{n}, \frac{\mathbf{y}_{n}-\mathbf{y}_{n-1}}{h}\right)=0
$$


where $h=t_{n}-t_{n-1}$. The resulting system of nonlinear equations for $\mathbf{y}_{n}$ at each time step is then usually solved by Newton's method (Brenan, Campbell and Petzold, 1996). The $k$-step (constant stepsize) BDF consists of replacing $\mathbf{y}^{\prime}$ by the derivative of the polynomial which interpolates the computed solution at $k+1$ times $t_{n}, t_{n-1}, \ldots, t_{n-k}$, evaluated at $t_{n}$. This yields

$$
\mathbf{F}\left(t_{n}, \mathbf{y}_{n}, \frac{\rho \mathbf{y}_{n}}{h}\right)=0
$$

where $\rho \mathbf{y}_{n}=\sum \alpha_{i} y_{n-i}$ and $\alpha_{i}, i=1,2, \ldots, k$ are the coefficients of the BDF method.

It is convenient to discuss the convergence of BDF method applied to fullyimplicit index-one systems.

Theorem 1 (Brenan et al. 1996) Let $\mathbf{F}\left(t, \mathbf{y}, \mathbf{y}^{\prime}\right)=0$ a uniform index one DAE on an interval $I=\left[t_{0}, t_{0}+T\right]$. Then the numerical solution of $\mathbf{F}\left(t, \mathbf{y}, \mathbf{y}^{\prime}\right)=0$ by the $k$-step BDF with fixed stepsize $h$ for $k<7$ converges to $O\left(h^{k}\right)$ if all initial values are correct to $O\left(h^{k}\right)$ accuracy and if the Newton iteration on each step is solved to $O\left(h^{k+1}\right)$ accuracy.

Thus we can conclude that the DAE under study can be solved with a code based on the BDF method. Popular DAE solution codes based on BDFs are DASSL (Brenan et al., 1996) and LSODI (Brenan et al., 1996). Here, the ode15s code of the MatLAB ODE suite is used. It was chosen because MatLab is one of the most widely used problem-solving environments. The ode15s code is based on a variant of the BDFs called Numerical Differentiation Formulas (NDFs). For further information about ode15s, see (Shampine, Reichelt and Kierzenka, 1999).

\section{Simulation Results}

The discretization grid was selected taking into account the operation of the reactor and the regions of interest in the particle size spectrum. For example, it is advisable to have a fine grid in the region corresponding to the particles leaving the reactor. For this reason, the grid was divided into two regions: fine particles, corresponding to the sizes of particles leaving the reactor, and coarse particles. The total number of intervals are thus split in two, half of the intervals corresponding to the region of fines and half of the intervals corresponding to the region of coarse particles. Finally, a linear grid is used in both regions. Figure 3 shows a sketch of such a discretization grid. The number of intervals in the grid has been chosen by running simulations for different number of intervals and selecting the minimum number of intervals that provides the same results as finer grids, based on visual inspection of the results.

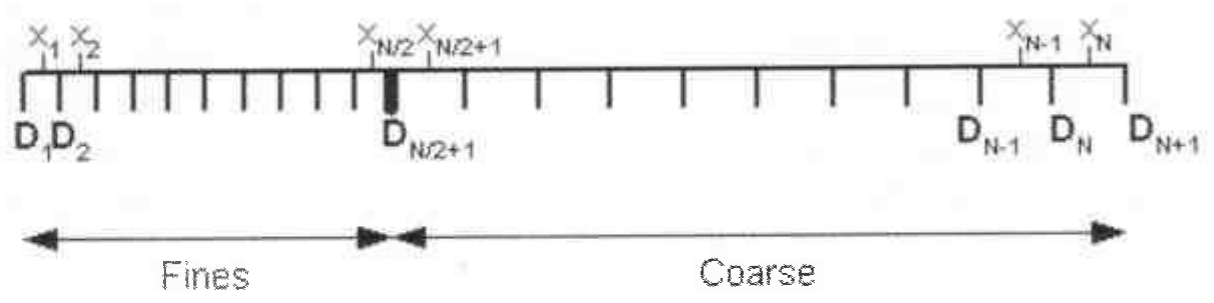

Figure 3. Discretization grid. 
Simulations have been carried out with initial conditions and inputs in the range of values of the real reactor. The system is simulated until it reaches steady-state as regards PSD and then the response of the system to a step change in one of the inputs is simulated. Note that scaled variables are used in all the figures.

Figures 4 and 5 show the evolution of the system after a step change in the FeSi feed, from the normal operating value to half this value. It can be seen that before the step change in FeSi feedrate, the volume of the reaction region $V_{1}(t)$ increases and never reaches steady-state, since more FeSi is fed than consumed in the disintegration. The open-loop operation is thus integrating. Once the FeSi mass input is halved, the volume in the reaction region continue to increase, but at a slower pace, as it could be expected. The volume of the storage region $V_{2}(t)$ also decreases, but at a slower pace after the step change. The change also affects the solid's particle fractions in both regions, decreasing in both cases, which is logical since less solid material is fed to the system whereas the fluid feed remains constant. Figure 4 also shows that the step change in the FeSi feed $\dot{M}_{\text {in }}$ results in an increase in Temperature $T(t)$. This result is reasonable since the heat exchange between the cold FeSi feed and the hot acid flowrate has been reduced. The cut size $D_{c}(t)$ does not vary much since the porosity in the storage region $\left(1-g_{2}(t)\right)$, which is the main factor determining its variation, has not been modified to a large extent. Finally, Figure 5 indicates that the PSD in the reaction region, and in the storage region are not greatly affected by a change in FeSi feedrate, as could be expected. Note that the
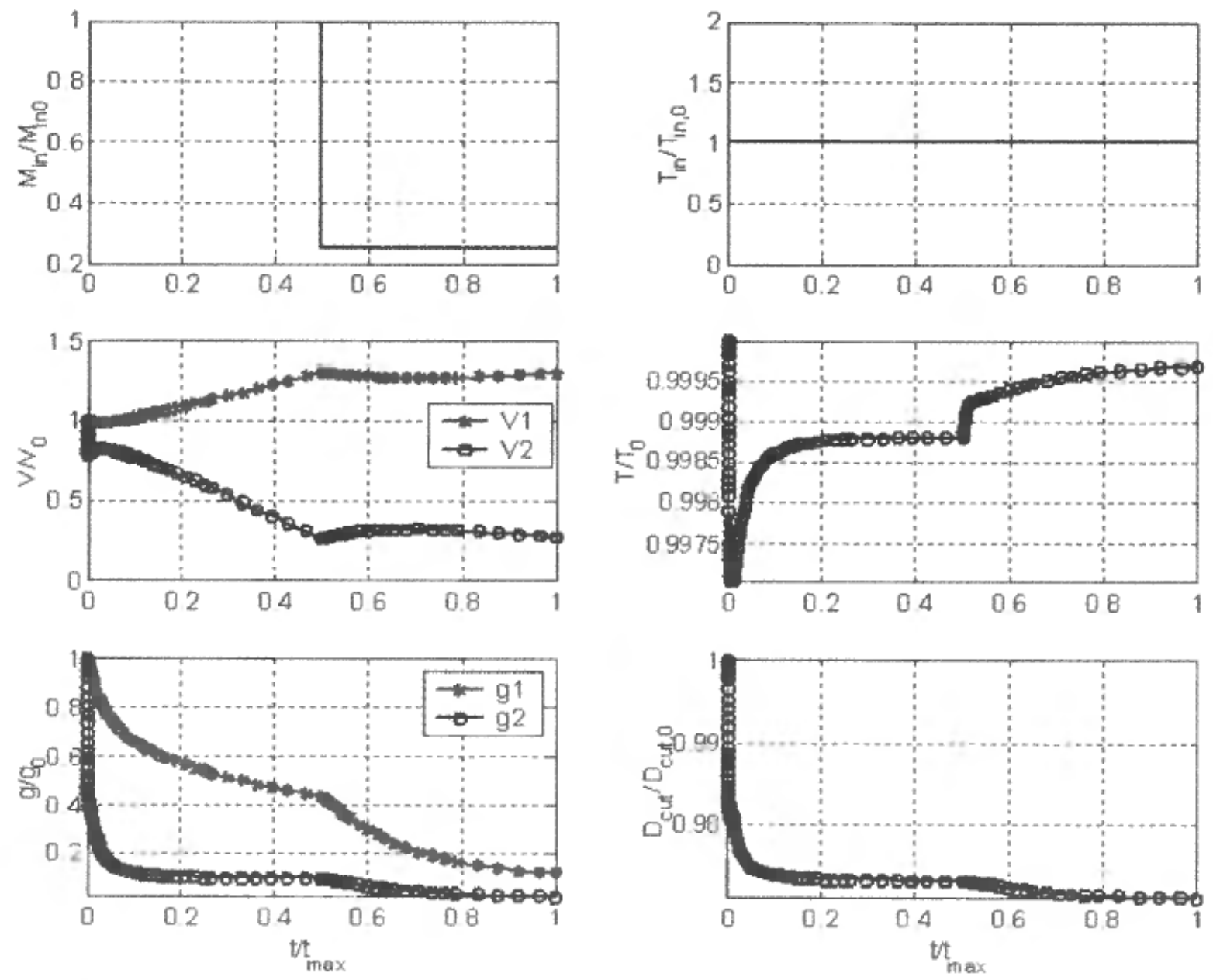

Figure 4. Time evolution of some states/outputs after a step change in FeSi feedrate. 


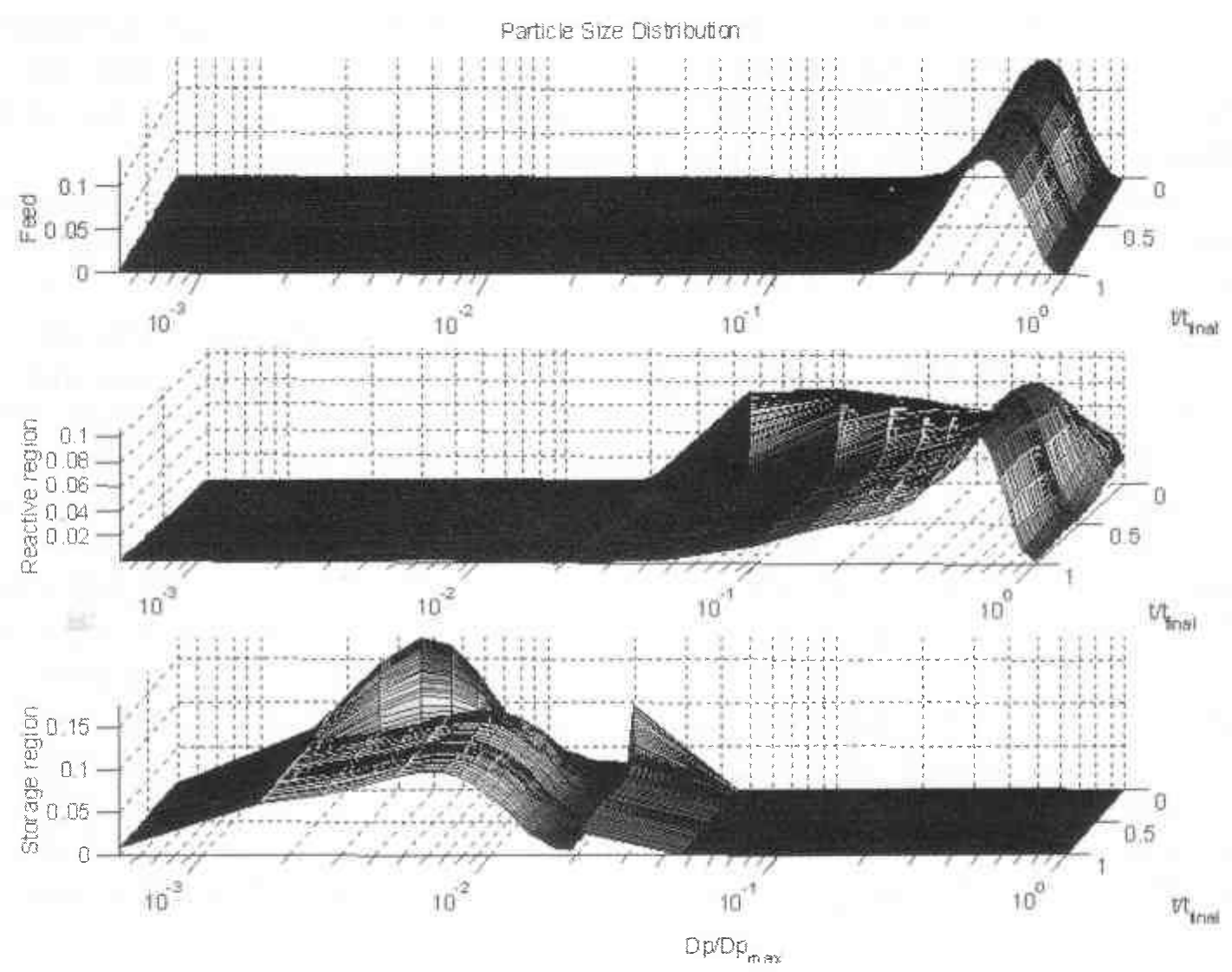

Figure 5. Time evolution of PSDs after a step change in FeSi feedrate.

PSD in the storage region differs greatly from the PSD in the reaction region, as we expected. In the reaction region coarse particles predominate, whereas in the storage region only fines are present.

Let us take a look now at the simulations of a step change in the temperature of the acid flow, which is increased by $2.5 \%$ with respect to the normal operating temperature. As can be observed in Figure 6, an increase in the acid temperature makes the disintegration proceed more quickly. This means that accumulation of FeSi in the reaction region is hindered and for this reason the volume in this region builds up more slowly. Similarly, the volume of the storage region decreases more slowly since more fines are produced now than prior to the step change. The same reasoning explains the observed trends for the solid's volume fraction within the two regions. Figure 6 shows that an equivalent increment in the reactor temperature is obtained and that the cut size evolves according to the evolution of the porosity of the storage region. The displacement of the PSD within the reaction region towards smaller sizes, and the change in PSD shape, as observed in Figure 7, confirm that a boost in inlet acid temperature enhances disintegration.

Figure 8 shows the result of a step change in the shape of the PSD of the FeSi feed. As it can be noticed, the PSD within the reaction region will experience a noticeable change, whereas the PSD in the storage region is not so much affected.

The simulation results are satisfactory and seem to match qualitatively with the knowledge of operation of the reactor. The new model resembles more closely the behavior of the industrial reactor than the previous model in (Dueñas and Lie, 2000). 

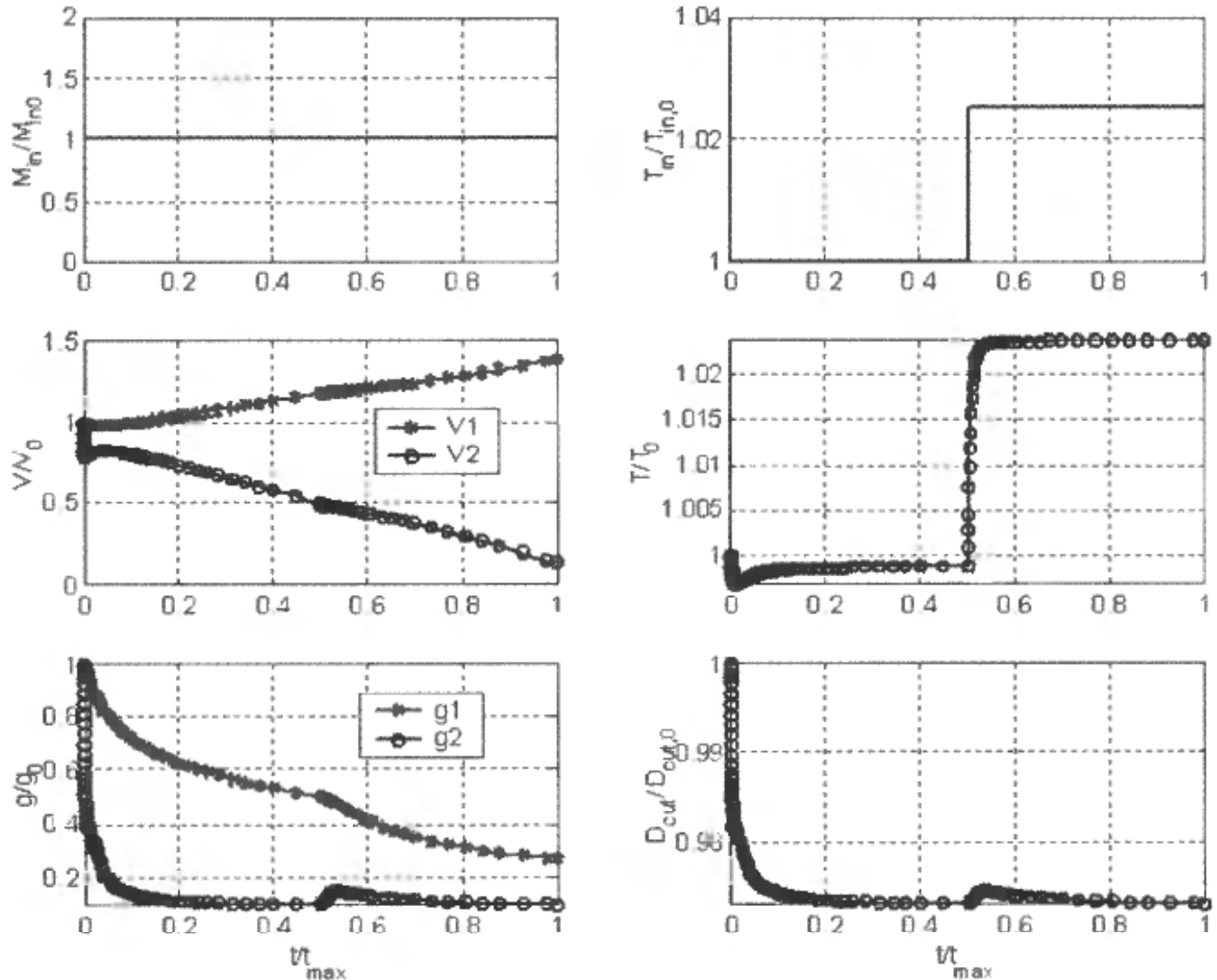

Figure 6. Time evolution of some states/outputs after a step change in inlet acid temperature.
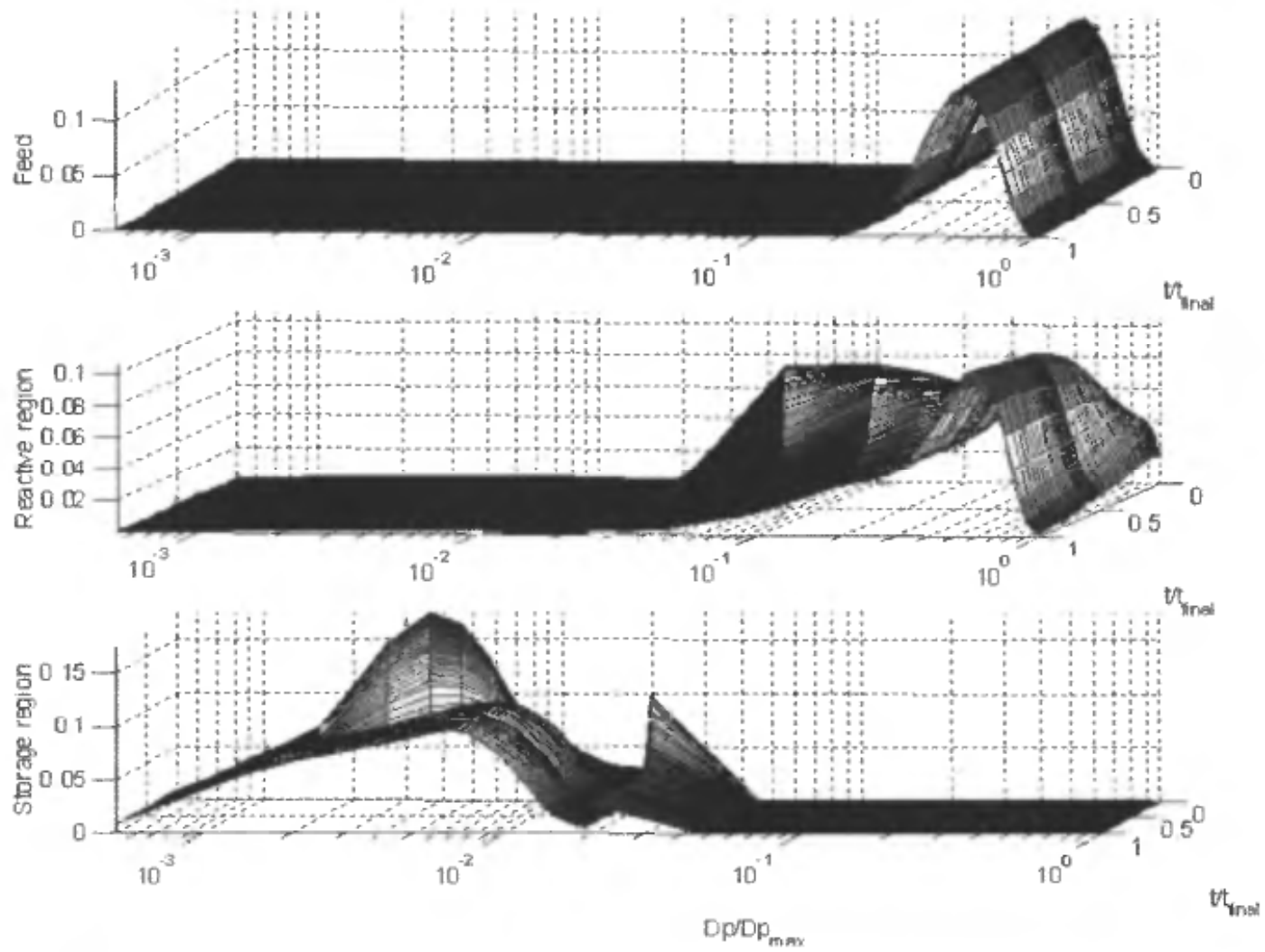

Figure 7. Time evolution of PSDs after a step change in inlet acid temperature. 

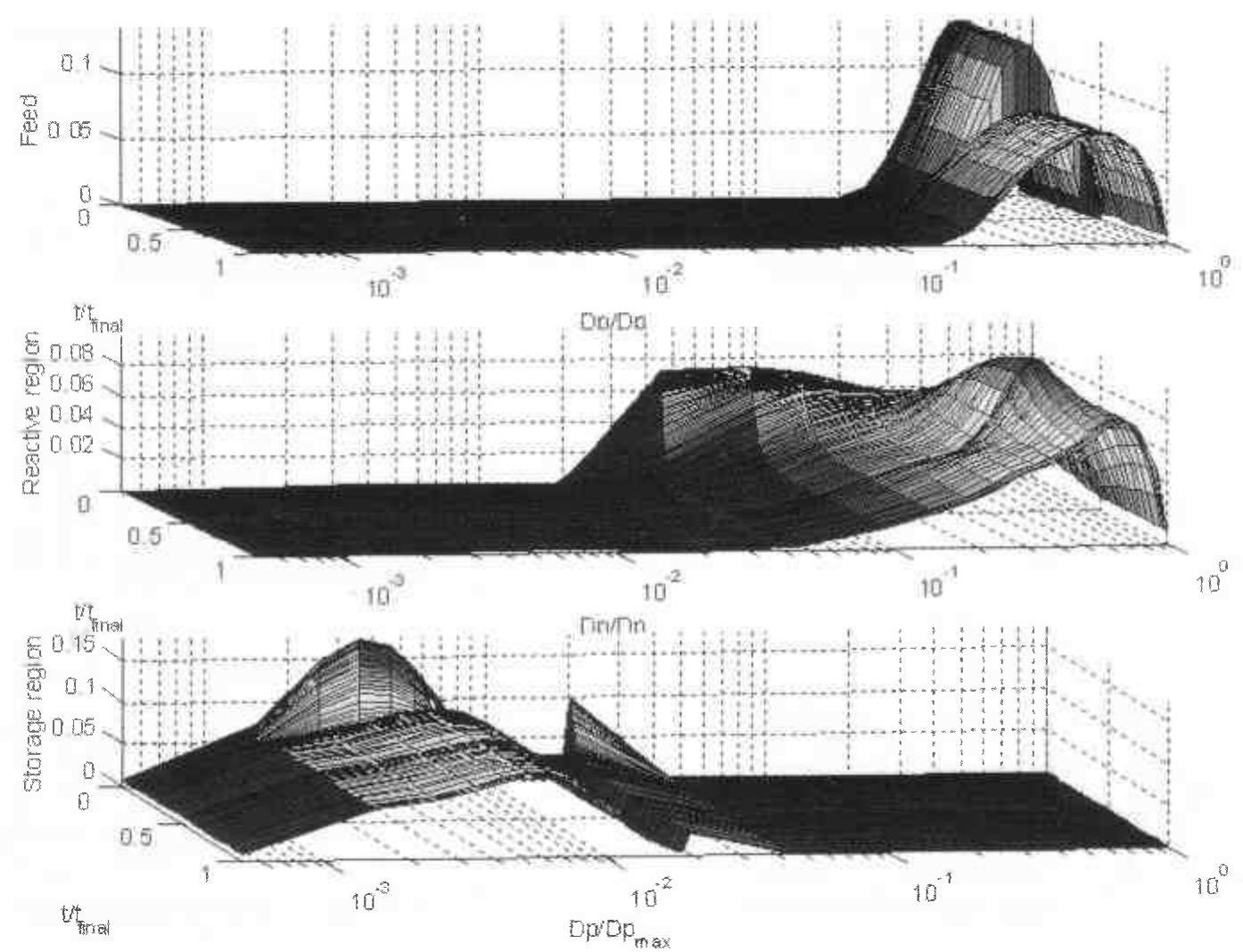

Figure 8. Time evolution of PSDs after a change in the PSDof FeSi feed.

\section{Conclusions}

The intention of this paper is to improve an existing dynamic model of the main reactor in the Silgrain ${ }^{\circledR}$ process for the production of $\mathrm{Si}$ from $\mathrm{FeSi}$ in order to resemble more closely the behavior of the real reactor. This requires the consideration of the momentum balance over the particles, since only the disintegrated material is displaced out from the reactor by the acid upflow, whereas the coarse material remains within the reactor until its disintegration. Furthermore, the energy balance has to be included since temperature plays an important role in the disintegration. Finally, a model for the disintegration terms based on laboratory experiments has been used.

The extended model consists of 1 integrodifferential equation, 4 implicit ordinary differential equations, 8 algebraic equations and 3 integral equations. The solution of such a difficult system of equations is approached here by using the discretization method proposed by Kumar and Ramkrishna (1996). The discretized system turns out to be a DAE system. DAEs are not ODEs and difficulties can be encountered when using numerical methods to solve them. Thus, DAEs require analysis and characterization and may require reformulation before considering its numerical solution. Such analysis is carried out here. It is concluded that the index of the system may vary with time and the operating conditions, but that for the normal range of operating conditions the DAE is implicit index-one and no reformulation is necessary. Attention is also given to the determination of consistent initial conditions since this is a key issue when solving DAEs. Particularly challenging is the selection of initial values for the discrete number-density PSD functions per unit working volume. The need of scaling the state variables and the system equations is also 
discussed. The analysis of the DAE system concludes with the selection of a numerical method to solve the system. A method based on the BDFs is appropriate for this system and the ode15s code of the MATLAB ODE suite, which is based on a variant of the BDFs called NDFs, is selected here.

The model is thus implemented in MATLAB and a simulation analysis is carried out to test the model. The simulation results are satisfactory and seem to match qualitatively with the known operation of the reactor.

To sum up, the main contributions of this paper refer to the following research areas:

- The use of the macroscopic PBE for modeling particulate processes. Previous researchers have made use of the assumption of complete-mixing for the PSD. We make a more general use of the macroscopic population equation. The outflow PSD in not longer assumed to be equal to the PSD within the reactor, but both PSDs are related by considering the momentum balance over the particles. Another contribution in the modeling area is the suggested functions for the disintegration terms, which have been obtained after experimental results at laboratory scale.

- The use and solution of DAE systems. DAEs are challenging and require a more thorough analysis than other mathematical systems. Here we present a complete example of an analysis of a DAE system. The determination of the index of the system, of consistent initial conditions and of the method of solution were the main issues in the analysis.

Further work will include a comparison of the simulation results with experimental data from the industrial reactor. Also, the model is expected to be used to design advanced control systems which may lead to an improvement in the operation and yield of the reactor. Passivity-based control and predictive control are two of the model-based control techniques in which the model presented here could be used.

\section{Acknowledgments}

The authors would like to thank Prof. Lorentz T. Biegler (Carnegie Mellon University, Pittsburgh, USA) for his useful advice regarding numerical solution of the model. We also want to acknowledge the help we received from Birte Skofteland, Anne Grete Forwald, Einar Andersen and the other employees at Elkem's Research Centre (Kristiansand, Norway) during the laboratory work that was carried out in this centre. The work of Marta Dueñas Díez is financially supported by the Research Council of Norway (project number 142994/432). Additional support has been obtained from Elkem ASA in relation to the case study.

\section{Notation}

Roman Symbols

\begin{tabular}{lll}
\hline Symbol & Definition & Units \\
\hline$a\left(D_{p}, T\right)$ & Particle breakup frequency function & $\mathrm{s}^{-1}$ \\
$b\left(D_{p}, y\right)$ & Birth probability distribution function & $\mathrm{m}^{-1}$ \\
$B$ & Particle birth rate & $1 / \mathrm{s} \mathrm{m}^{4}$ \\
$C p$ & Heat Capacity & $\mathrm{kcal} / \mathrm{kg} \mathrm{K}^{-}$ \\
$D$ & Particle death rate & $1 / \mathrm{s} \mathrm{m}^{4}$
\end{tabular}




\begin{tabular}{lll}
$D_{\text {cut }}$ & Cut size & $\mathrm{m}$ \\
$D_{p}$ & Particle diameter & $\mathrm{m}$ \\
$f$ & PSD as mass probability distribution function & $\mathrm{m}^{-1}$ \\
$g$ & solid's volume fraction & - \\
$g_{r}$ & Gravity acceleration & $\mathrm{m} / \mathrm{s}^{2}$ \\
$N$ & Number of intervals & - \\
$\dot{M}$ & Mass Flowrate & $\mathrm{kg} / \mathrm{s}$ \\
$q$ & Volumetric slurry flow rate & $\mathrm{m}^{3} / \mathrm{s}$ \\
$Q$ & Heat loss to the surroundings & $\mathrm{kcal} / \mathrm{s}$ \\
$t$ & Time & $\mathrm{s}$ \\
$T$ & Temperature & $\mathrm{K}$ \\
$u_{e t}$ & Superficial velocity & $\mathrm{m} / \mathrm{s}$ \\
$u_{o}$ & Actual fluid velocity & $\mathrm{m} / \mathrm{s}$ \\
$V$ & Working volume of the reactor & $\mathrm{m}$ \\
$x$ & Representative diameter & $\mathrm{m}$ \\
$y$ & Mother particle diameter & $\mathrm{m}$ \\
\hline
\end{tabular}

Greek Symbols

\begin{tabular}{lll}
\hline Symbol & Definition & Units \\
\hline$\delta$ & Delta Dirac function & - \\
$\eta$ & Average number of daughter particles & - \\
$\rho$ & Density & $\mathrm{kg} / \mathrm{m}^{3}$ \\
$\rho C_{p}$ & Volumetric Heat Capacity & $\mathrm{kcal} / \mathrm{m}^{3} \mathrm{~K}$ \\
$\phi$ & Discrete particle size number-density distribution & $\mathrm{m}^{-3}$ \\
$\psi$ & Continuous particle size number-density distribution & $\mathrm{m}^{-4}$ \\
\hline
\end{tabular}

Abbreviations

\begin{tabular}{ll}
\hline BDF & Backward Differentiation Formulas \\
DAE & Differential and Algebraic Equations \\
NDF & Numerical Differentiation Formulas \\
ODE & Ordinary Differential Equations \\
PBE & Population Balance Equation \\
PSD & Particle Size Distribution
\end{tabular}

Subscripts

\begin{tabular}{ll}
\hline Symbol & Definition \\
\hline in & Relative to the inlet to the reactor \\
inter & Relative to the flow from the reaction to the storage region \\
$\max$ & maximum \\
$\min$ & minimum \\
$f$ & Relative to the liquid \\
$p$ & Relative to particle \\
out & Relative to the outflow from the reactor \\
1 & Relative to the reaction region of the reactor \\
2 & Relative to the storage region of the reactor
\end{tabular}

\section{REFERENCES}

AAS, H. (1971). The silgrain process: Silicon metal from 90 ferrosilicon, in Light Metals 1971: Proceedings of Symposia 100th AIME Annual Meeting, number A71-47, pp. 650-667.

Brenan, K. E., Campbell, S. L. and Petzold, L. R. (1996). Numerical Solution of InitialValue Problems in Differential-Algebraic Equations, SIAM Society for Industrial and Applied Mathematics. 
Chen, Z., Prüss, J. and Warbecke, H. (1998). A population balance model for disperse systems: Drop size distribution in emulsion, Chemical Engineering Science 53(5), 1059 1066.

Coulson, J. and Richardson, J. (1978). Chemical Engineering. Fluid Flow, Heat Transfer and Mass Transfer, Vol. 1, Pergamon Press, Inc.

DuEÑAs, M. and LIE. B. (2000). Modelling and simulation of a hydrometallurgical leaching reactor, in B. ElmegaArd, N. HoubaK, A. JakobSEn and F. J. WAGNer, eds, Proceedings SIMS 2000, SIMS-Scandinavian Simulation Society and Technical University of Denmark, Lyngby (Denmark), pp. 199-225.

HerbST, J. (1979). Rate processes in multiparticle metallurgical systems, in H. SoHN and M. Wadsworth, eds. Rate Processes in Extractive Metallurgy, Plenum Press, pp. 53-112.

Herbst, J. and AsIHENE, S. (1993). Modelling and simulation of hydrometallurgical processes using the population balance approach, in V. PAPANGelakis and G. Demopoulos, eds, Modelling, Simulation and Control of Hydrometallurgical Processes. Proceedings of the 32nd Annual Conference of Metallurgists of CIM, Canadian Institute of Mining, metallurgy and petroleum. Quebec, pp. 3-44.

Hulburt, H. M. and Katz, S. (1964). Some problems in particle technology-A statistical mechanical formulation, Chemical Engineering Science 19, 555-574.

KAPUR, P. (1995). Particle population balance in granulation of iron ores by an auto-layering mode, in S. Mehrotra and R. Shekhar, eds, Mineral Processing. Recent Advances and Future Trends., Allied Publishers Ltd, pp. 703-717.

Kumar, S. and Ramkrishna, D. (1996), On the solution of population balance equations by discretization-1. A fixed pivot technique, Chemical Engineering Science 51(8), $1311-1332$.

Lefkopoulos, A. and StadherR, M. A. (1993). Index analysis of unsteady-state chemical process systems-I. An algorithm for problem formulation, Computers Chem. Engng. 17(4), 399-413.

Margarido, F., Martins, J. P., Figueiredo, M. O. and Bastos, M. H. (1993). Kinetics of acid leaching refining of an industrial fe-si alloy, Hydrometallurgy 34, 1-11.

Petzold, L. R. (1985). Differential-algebraic equations are not ODEs, SIAM J. Sci. Stat. Compt. 3(3), 49-95.

Ramkrishna, D. (1985). The status of population balances, Reviews in Chemical Engineering 3, 49-95.

RANDOLPH, A. (1964). A population balance for countable entities, The Canadian Journal of Chemical engineering 42, 280-281.

Randolph, A. D. and Larson, M. A. (1988). Theory of Particulate Processes. Analysis and Techniques of Continuous Crystallization, second edn, Academic Press, Inc.

Rubisov, D. H. and Papangelakis, V. G. (1996a). Mathematical modelling of the transient behaviour of CSTRs with reactive particulates: Part I-The population balance framework, The Canadian Journal of Chemical Engineering 74, 353-362.

Rubisov, D. H. and Papangelakis, V. G. (1996b). Mathematical modelling of the transient behaviour of CSTRs with reactive particulates: Part 2-Application to pyrite pressure oxidation, The Canadian Journal of Chemical Engineering 74, 363-371.

Rubisov, D. and PAPANGelakis, V. (1995). Model-based analysis of pressure oxidation autoclave behaviour during process upsets, Hydrometallurgy 39, 377-389.

Shampine, L. F., Reichelt, M. W. and Kierzenka, J. A. (1999). Solving index-1 DAEs in matlab and simulink, SIAM Review 41(3), 538-552. 